\title{
Surgical Cost Increase With Age in Emergency But Not Elective General Surgery
}

\section{Eamer $\mathrm{G}^{1}$, Brisebois $\mathrm{R}^{1}$, Clement $\mathrm{F}^{2}$, Khadaroo $\mathrm{RG}^{1}$}

${ }^{1}$ University of Alberta, ${ }^{2}$ University of Calgary

\section{Background}

- The Canadian population is aging and are increasingly in need of surgical interventions.

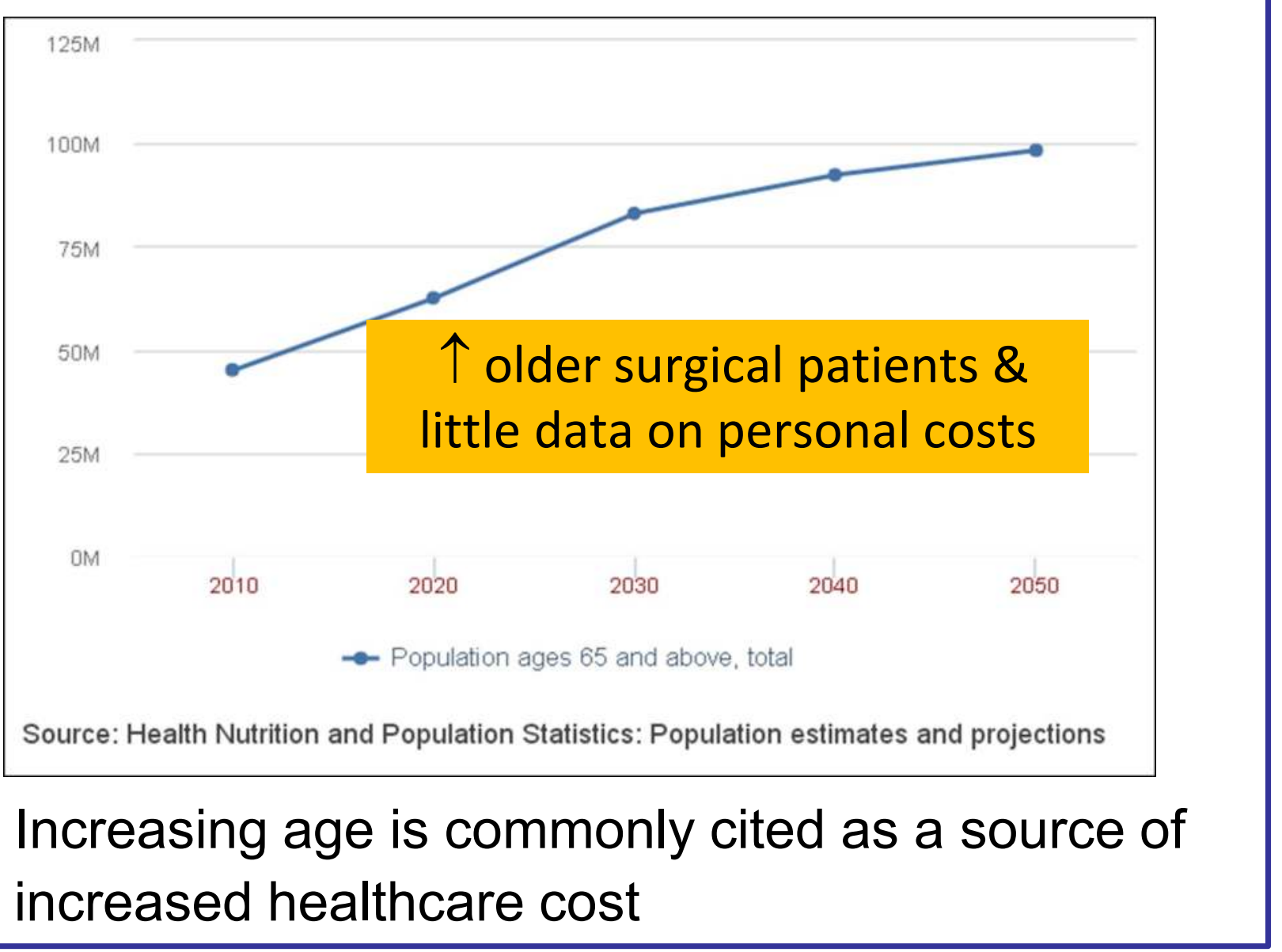

\section{Objective}

- To assess the effect of age on surgical costs in a large cohort of patients undergoing both elective and emergency surgery

\section{Methods}

- Retrospective analysis of general surgical inpatient costs over two fiscal years of four hospitals.

- Cost and number of procedures were reported by age, procedure, hospital, cost category, and surgical urgency.

- Costs were compared between surgical risk profile, urgency and age.

- Cost differences of $10 \%$ or greater were considered clinically significant.

\section{Results}

- 12,070 inpatient surgical procedures ( $84 \%$ of all admissions in the region) were examined.

- The average cost was $\$ 4,351$ for scheduled admissions and $\$ 4,054$ for unscheduled admissions.

- Post-operative care costs drove the increased cost in older age groups

\begin{tabular}{|c|c|c|c|c|c|c|c|c|c|c|c|}
\hline \multirow{2}{*}{\multicolumn{2}{|c|}{$\begin{array}{l}\text { Age Category } \\
64 \text { and Under }\end{array}$}} & \multirow{2}{*}{$\begin{array}{l}\begin{array}{l}\text { Total } \\
\text { Cases }\end{array} \\
8451\end{array}$} & \multirow{2}{*}{$\begin{array}{l}\begin{array}{l}\text { Inpatient } \\
\text { cost }\end{array} \\
\$ 3,951\end{array}$} & \multicolumn{2}{|c|}{$\begin{array}{l}\text { Total } \\
\text { Scheduled } \\
\text { Cases }\end{array}$} & \multicolumn{2}{|c|}{$\begin{array}{l}\text { Inpatient Cost } \\
\text { Scheduled Cases }\end{array}$} & \multirow{2}{*}{$\begin{array}{l}\text { Total } \\
\text { Unscheduled } \\
\text { Cases } \\
4078\end{array}$} & \multicolumn{3}{|c|}{$\begin{array}{l}\text { Inpatient Cost } \\
\text { Unscheduled Cases }\end{array}$} \\
\hline & & & & \multicolumn{2}{|l|}{4373} & \multicolumn{2}{|c|}{4,422} & & 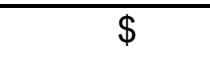 & & 3,447 \\
\hline \multicolumn{2}{|l|}{65 to 79} & 2863 & $\$ 4,794$ & 2021 & & \multicolumn{2}{|c|}{4,333} & 842 & $\$$ & & 5,902 \\
\hline \multicolumn{2}{|l|}{$80+$} & 756 & $\$ 5,105$ & 500 & & \multicolumn{2}{|c|}{3,815} & 256 & $\$$ & \multicolumn{2}{|r|}{7,622} \\
\hline Average & & 12070 & $\$ 4,224$ & 6894 & $\$$ & 4,35 & & 5176 & $\$$ & \multicolumn{2}{|r|}{4,054} \\
\hline \multirow{2}{*}{$\begin{array}{l}\text { Risk } \\
\text { category }\end{array}$} & \multirow{2}{*}{\multicolumn{2}{|c|}{ Age group }} & \multicolumn{3}{|c|}{ Overall } & \multicolumn{3}{|c|}{ Scheduled } & \multicolumn{3}{|c|}{ Unscheduled } \\
\hline & & & $\%$ change & $\mathrm{n}$ & $\mathrm{p}$ & $\%$ change & $\mathrm{n}$ & $\mathrm{p}$ & $\%$ change & $\mathrm{n}$ & $\mathrm{p}$ \\
\hline \multirow{3}{*}{ Low risk } & \multirow{2}{*}{\multicolumn{2}{|c|}{$\begin{array}{l}\text { Under } 65 \\
65 \text { to } 79\end{array}$}} & \multicolumn{3}{|c|}{1574} & \multicolumn{3}{|c|}{1258} & \multicolumn{3}{|c|}{316} \\
\hline & & & $-2.90 \%$ & 678 & 0.06 & $-8.80 \%$ & 621 & $<.001$ & $48.10 \%$ & 57 & $<.001$ \\
\hline & \multicolumn{2}{|c|}{$80+$} & $8.60 \%$ & 244 & $<.001$ & $-8.80 \%$ & 206 & $<.001$ & $115.30 \%$ & 38 & $<.001$ \\
\hline \multirow{3}{*}{$\begin{array}{l}\text { Moderate } \\
\text { risk }\end{array}$} & \multicolumn{2}{|c|}{ Under 65} & \multicolumn{3}{|c|}{3,497} & \multicolumn{3}{|c|}{760} & \multicolumn{3}{|c|}{2737} \\
\hline & 65 & 079 & $29.70 \%$ & 757 & $<.001$ & $2.50 \%$ & 414 & 0.052 & $36.80 \%$ & 343 & 0.045 \\
\hline & $80-$ & & $63.00 \%$ & 132 & $<.001$ & $1.50 \%$ & 56 & 0.94 & $103.30 \%$ & 76 & $<.001$ \\
\hline & Un & ler 65 & & 1,096 & & & 600 & & & 496 & \\
\hline High risk & 65 & o 79 & $9.70 \%$ & 679 & 0.019 & $2.60 \%$ & 424 & 0.6 & $22.60 \%$ & 255 & 0.04 \\
\hline & 80 & & $25.80 \%$ & 186 & 0.002 & $5.10 \%$ & 98 & 0.6 & $46.00 \%$ & 88 & $<.001$ \\
\hline
\end{tabular}

ADMISSION COST (CAD) BY AGE AND SURGICAL RISK

PROFILE FOR PLANNED AND UNPLANNED SURGERY

Unscheduled Under 65 years Unscheduled 65 to 79 years Unscheduled $80+$ years

- Scheduled Under $65 \quad$ Scheduled 65 to $79 \quad$ Scheduled*80+

\section{Discussion}

- Surgeons likely select for good surgical candidates in scheduled surgical cases.

- Older patients who would not tolerate surgery may be steered towards medical management or are being optimized for surgery before intervention.

- Frailty is more predictive of post-operative morbidity and mortality and may better predict cost.

- Improving outcomes and decreasing cost in unscheduled surgery requires novel approaches to care

- Comprehensive geriatric assessment may be one such tool and has been shown to decrease cost in hip fracture patients

\section{Conclusion}

- Surgical intervention in the elderly results in higher costs of care for only unscheduled cases. This is likely due to surgeons screening patients at risk of adverse outcomes.

- Innovative programs to improve outcomes in older unscheduled surgical patients may decrease cost

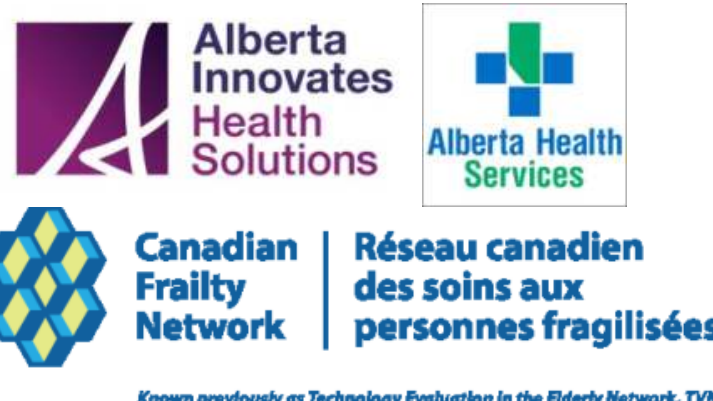

